Benjamin D. Singer

Franco R. D'Alessio

\section{Comment on Adamzik et al.: An increased alveolar CD4 + CD25 + Foxp3 + T-regulatory cell ratio in acute respiratory distress syndrome is associated with increased 30-day mortality}

Accepted: 4 July 2014

Published online: 17 July 2014

(C) Springer-Verlag Berlin Heidelberg and ESICM 2014

Dear Editor,

Adamzik et al. [1] reported that an elevated ratio of $\mathrm{CD} 4{ }^{+} \mathrm{CD} 25^{+}$

Foxp $3^{+}$regulatory $\mathrm{T}$ cells (Tregs) to overall $\mathrm{CD}^{+}{ }^{+}$cells in bronchoalveolar lavage (BAL) fluid portends a poor outcome for ARDS patients. A cellular biomarker able to provide prognostic information would be useful for ICU decision-making. However, we wish to highlight that the functional significance of alveolar Tregs remains undefined in ARDS.

Our group has shown in mouse models that Tregs - a lymphocyte subset that attenuates immune system activation and promotes repair in damaged tissues-resolve experimental lung injury via pro-repair effects on macrophage function and neutrophil efferocytosis [2], epithelial regeneration [3], and fibroproliferation [4]. It is unclear from the authors' data, obtained at a single time point, whether Tregs were actually pathogenic or if they accumulated as a pro-repair response to parenchymal damage. We hypothesize that severe lung inflammation, rather than the Tregs themselves, mediated the observed increase in mortality. A few findings call into question the study's conclusions:

1. Validated illness severity markers such as the SAPS II and lung injury scores did not predict mortality in their cohort. Additionally, in the authors' quantification of Treg-to-CD4 ${ }^{+}$cell ratio there was one extreme outlier in the nonsurvivor group. Would the authors' conclusions hold if this data point were excluded in a sensitivity analysis?

2. Study group characteristics likely affected the results. All ARDS subjects and an unknown proportion of non-ARDS controls received glucocorticoids, which probably altered lymphocyte population features. The fact that control subjects had active malignancies may also have skewed the findings, as Tregs are known to play a major role in cancer biology.

3. Phenotypically, $\mathrm{CD} 4^{+} \mathrm{CD} 25^{+}$Foxp $^{+}$status is insufficient to identify human Tregs and may include newly activated effector $\mathrm{T}$ cells. Other markers including CD3, CD127, and CD45 isoforms are usually advocated to increase specificity when analyzing human T lymphocyte populations [5]. A more specific Treg definition could have changed the conclusions of the study.

4. Finally, in addition to the reported ratio value, was the absolute Treg number per unit volume of BAL fluid different between the groups?

Many questions remain regarding the impact of alveolar Tregs in ARDS. Foremost, if these potent immunoregulatory cells are indeed lung inflammation markers, what are the mechanisms that limit their prorepair activity following severe lung injury? Further characterization of alveolar Treg phenotype and function during resolution of human lung injury will help determine their usefulness as a biomarker and potential therapeutic target for ARDS.

Conflicts of interest This manuscript was prepared in the absence of any commercial or financial relationships that could be construed as a potential conflict of interest.

\section{References}

1. Adamzik M, Broll J, Steinmann J et al (2013) An increased alveolar CD4 + CD25 + Foxp3 + T-regulatory cell ratio in acute respiratory distress syndrome is associated with increased 30-day mortality. Intensive Care Med 39:1743-1751. doi:10.1007/s00134013-3036-3

2. D'Alessio FR, Tsushima K, Aggarwal NR et al (2009) CD4 + CD25 + Foxp3 + Tregs resolve experimental lung injury in mice and are present in humans with acute lung injury. J Clin Invest 119:2898-2913. doi:10.1172/JCI36498

3. Mock JR, Garibaldi BT, Aggarwal NR et al (2014) Foxp3(+) regulatory T cells promote lung epithelial proliferation. Mucosal Immunol. doi: 10.1038/mi.2014.33

4. Garibaldi BT, D'Alessio FR, Mock JR et al (2013) Regulatory T cells reduce acute lung injury fibroproliferation by decreasing fibrocyte recruitment. Am J Respir Cell Mol Biol 48:35-43. doi: 10.1165/rcmb.2012-0198OC

5. Singer BD, King LS, D'Alessio FR (2014) Regulatory T cells as immunotherapy. Front Immunol 5:46. doi:10.3389/fimmu.2014.00046

B. D. Singer · F. R. D’Alessio (『)

Division of Pulmonary and Critical Care Medicine, Johns Hopkins University,

5501 Hopkins Bayview Circle, 4th Floor, Baltimore, MD 21224, USA

e-mail: fdaless2@jhmi.edu

Tel.: 410-550-1282 